\title{
High thrombotic risk increases adverse clinical events up to 5 years after acute myocardial infarction. A nationwide retrospective cohort study
}

\author{
Gian Francesco Mureddu',2, Cesare Greco ${ }^{1}$, Stefano Rosato ${ }^{3}$, Paola D'Errigo ${ }^{3}$, Leonardo De Luca², \\ Gabriella Badoni $^{3}$, Pompilio Faggiano ${ }^{4,5}$, Fulvia Seccareccia ${ }^{3}$ \\ ${ }^{1}$ Cardiology and Cardiovascular Rehabilitation Unit, Department of Medicine, S. Giovanni-Addolorata Hospital, \\ Rome; ${ }^{2}$ Interventional Cardiology Unit, S. Giovanni Evangelista Hospital, Tivoli, Rome; ${ }^{3}$ National Center for Global \\ Health, Istituto Superiore di Sanità, Rome; ${ }^{4}$ Cardiology Division, Spedali Civili and University of Brescia; ${ }^{5}$ Italian \\ Association of Preventive Cardiology and Rehabilitation, Italy
}

\begin{abstract}
The risk of recurrent events among survivors of acute myocardial infarction (AMI) is understudied. The aim of this analysis was to investigate the role of residual high thrombotic risk (HTR) as a predictor of recurrent in-hospital events after AMI. This retrospective cohort study included 186,646 patients admitted with AMI from 2009 to 2010 in all Italian hospitals who were alive 30 days after the index event. HTR was defined as at least one of the following in the 5 years preceding AMI: previous myocardial infarction, ischemic stroke/other vascular disease, type 2 diabetes mellitus, renal failure. Risk adjustment was performed in all multivariate survival analyses. Rates of major cardiac and cerebrovascular events (MACCE) within the following 5 years were calculated in both patients without fatal readmissions at 30 days and in those free from in-hospital MACCE at 1 year from the index hos-
\end{abstract}

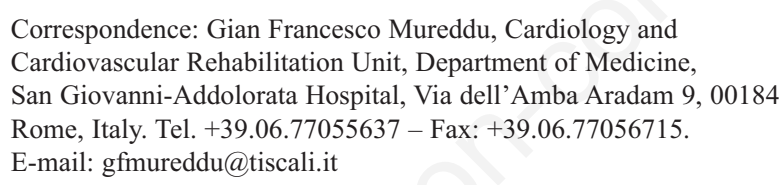

Key words: Acute myocardial infarction; high thrombotic risk, MACCE; long-term mortality; trend.

Conflict of interest: The authors declare no conflict of interest.

Acknowledgements: This study was carried out as part of a scientific collaboration agreement between the Italian Association for Cardiovascular Prevention, Rehabilitation and Epidemiology (IACPR) and the Italian National Institute of Health (Istituto Superiore di Sanità).

Received for publication: 9 September 2019

Accepted for publication: 10 October 2019.

${ }^{\circ}$ Copyright: the Author(s), 2019

Licensee PAGEPress, Italy

Monaldi Archives for Chest Disease 2019; 89:1166

doi: 10.4081/monaldi.2019.1166

This article is distributed under the terms of the Creative Commons Attribution Noncommercial License (by-nc 4.0) which permits any noncommercial use, distribution, and reproduction in any medium, provided the original author(s) and source are credited. pitalization. The overall 5-year risk of MACCE was higher in patients with HTR than in those without HTR, in both survivors at 30 days [hazard ratio (HR), 1.49; 95\% confidence interval (CI), $1.45-1.52 ; \mathrm{p}<0.0001]$ and in those free from MACCE at 1 year (HR, 1.46; 95\% CI, 1.41-1.51; $\mathrm{p}<0.0001$ ). The risk of recurrent MACCE increased in the first 18 months after AMI (HR, 1.49) and then remained stable over 5 years. The risk of MACCE after an AMI endures over 5 years in patients with HTR. This is also true for patients who did not have any new cardiovascular event in the first year after an AMI. All patients with HTR should be identified and addressed to intensive preventive care strategies.

\section{Introduction}

Despite the improvement in management of acute myocardial infarction (AMI), long-term risk of fatal and non-fatal cardiovascular events is still increasing over time [1-11]. Accordingly, several studies investigated the long-term effect of different secondary prevention strategies on prognosis of post- myocardial infarction (MI) patients [12-15]. However, the time course of residual ischemic risk remains unclear, particularly for patients who did not have any new cardiovascular event during the first year after AMI and then could apparently be considered not at high risk. Aim of this study is to investigate the prognostic significance of residual high thrombotic risk (HTR) features in a large population surviving AMI in order to identify patients who could potentially benefit from more intensive secondary prevention care strategies [1].

\section{Patients and Methods}

\section{Study design}

This is a retrospective cohort study based on data from the Italian National Registry of Hospital Discharge Records (ItHDR), the administrative database including all Italian hospital admissions and discharges. Given the retrospective nature of the study and the use of anonymized patient data, ethical approval was not required.

\section{Study population}

The ItHDR, including all records over two years, from January $1^{\text {st }} 2009$ to December $31^{\text {st }} 2010$, was used to identify the study population. ItHDR from January ${ }^{\text {st }} 2004$ to December $31^{\text {st }} 2008$ and 
ItHDR from January $1^{\text {st }} 2011$ to December $31^{\text {st }} 2015$ was then used to detect previous and subsequent hospitalizations for the study population (Hospital Discharge Records database -Italian Ministry of Health). All hospital discharge records of patients aged 18-100 years, resident in Italy, and reporting a primary diagnosis of AMI [International Classification of Disease, $9^{\text {th }}$ Revision, Clinical Modification (ICD 9 CM) 410] or a secondary diagnosis of AMI with any concomitant complication within the primary diagnosis (ICD-9-CM codes 411, 413, 414, 426, 427, 428, 423.0, 429.5, 429.6, 429.71, 429.79, 429.81, 518.4, 518.81, 780.01, 780.2, 785.51, 799.1, 997.02, and 998.2) were selected (Outcomes Evaluation National Program). Information on patient age, gender, risk factors, and comorbidities was also retrieved. ICD codes used to define outcomes and comorbidities have been reported in Table S1.

Patients discharged within $48 \mathrm{~h}$ from admission and those with a previous AMI admission within 30 days from the index admission were excluded to minimize the inclusion of false diagnosis of AMI and multiple admissions due to the same event [16,17]. For the purpose of this study, only patients without fatal in-hospital events 30 days after the index event entered into the current analysis (SURV30). Moreover, all patients free from in-hospital major adverse cardiac and cerebrovascular events (MACCE) resulting in hospitalization within the first 1 year from the index AMI, which is currently considered the period of greatest exposure to risk of recurrences, were also analyzed (NO-MACCE1Y). According to diagnostic criteria used in previous epidemiological [11] and clinical studies $[12,13]$ patients were classified as having high thrombotic risk (HTR) on the basis of the presence of at least one of the following characteristics recorded either in the index admission or in the previous 5-year hospitalizations: previous AMI, previous ischemic stroke (IS), other vascular disease, type 2 diabetes mellitus, or renal failure (National Kidney Foundation stage $\geq 3$ ). Following the same methodology, information on patient age, gender, risk factors, and comorbidities was also retrieved. Figure 1 shows the flowchart of the study.

\section{Outcomes}

MACCE (i.e., death from any cause, AMI or IS) and recurrent AMI within 5 years were considered as the main adverse outcomes. They were evaluated both in SURV30 and in NOMACCE1Y.

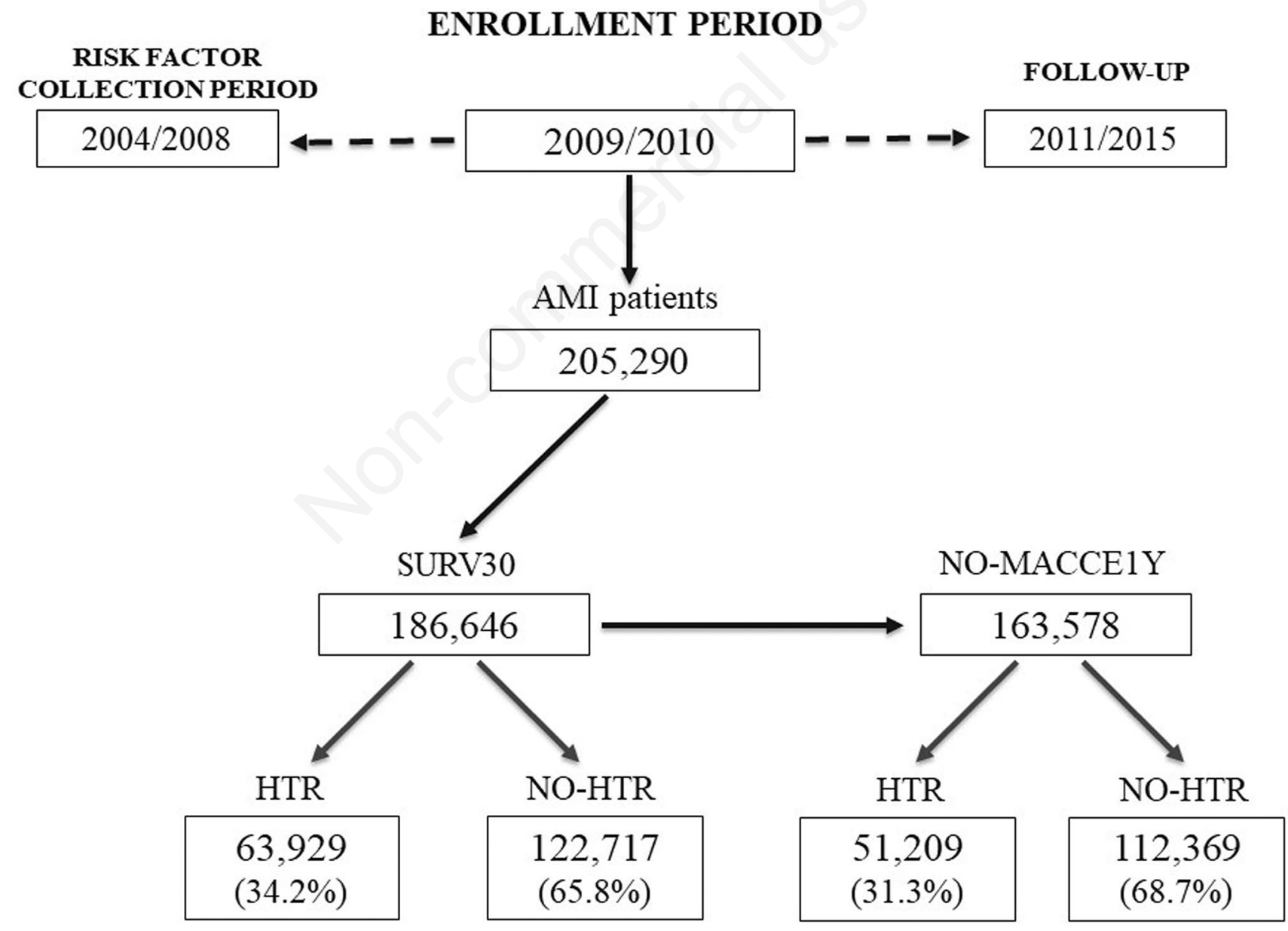

Figure 1. Flowchart of the study. The study population was identified from the Italian National Registry of Hospital Discharge Records (ItHDR) including all hospital discharge records of patients aged 18-100 years, resident in Italy, and reporting a primary diagnosis of AMI, over two years, from January $1^{\text {st }} 2009$ to December $31^{\text {st }} 2010$. The previous (2004-2008) and following (2011-2015) four years were used to detect previous and subsequent hospitalizations (Hospital Discharge Records database). 


\section{Statistical analysis}

All the statistical analyses were performed separately for SURV30 and NO-MACCE1Y. Prevalence of risk factors and comorbidities were presented as counts and percentages; age was expressed as the mean $\pm \mathrm{SD}$. Time to event distribution was analyzed according to the event type (MACCE or recurrent AMI). A Cox regression model was used for analysis of time to MACCE. Proportional hazards and time-dependent effects were checked using Shoenfeld residuals and the Kolmogorov-Smirnov test. Fine and Gray models were used for competing risk analysis of time to recurrent AMI, with death as the competing risk. Selection of variables was performed by stepwise multiple regression with the Akaike information criterion for selection. Cumulative incidence function (CIF) of MACCE and recurrent AMI was plotted separately for patients with HTR and those without HTR. The effect of covariates on the CIF was also evaluated using a proportional hazard model where the effect of HTR was allowed for time-varying over the 5 years of follow-up; the effect of all the other covariates was considered constant. Two-sided statistics were performed with a significance level of 0.05 . SAS version 9.2 and R 3.0.3 software [18] was used for all analyses.

\section{Results}

Of the 205,290 patients admitted for AMI to an Italian hospital in $2009-2010,186,646$ patients $(90.9 \%)$ did not have fatal in-hos- pital events 30 days after the event (SURV30); furthermore, 163,578 out of $186,646(87.6 \%)$ were free from in-hospital MACCE at 1 year (NO-MACCE1Y). Of the SURV30 group, $63,929(34.2 \%)$ were identified as having HTR; of the NOMACCE1Y group, the HTR condition was detected in 51,209 (31.3\%) patients (Figure 1).

The general characteristics as well as the prevalence and distribution of risk factors and main comorbidities in the SURV30 and NO-MACCE1Y groups are shown in Table 1. More than half of patients underwent percutaneous coronary intervention (PCI) at the index hospitalization (53.6\% of the SURV30 group and 56\% of the NO-MACCE1Y group); previous PCI was detected in $11 \%$ of patients in both groups. The proportion of patients with previous stable coronary heart disease was about $20 \%$ in both groups (20.9\% and $19 \%$, respectively).

A previous AMI was the most prevalent ischemic risk factor in both SURV30 and in NO-MACCE1Y (17.3\% and 15.8\%, respectively). The other most prevalent risk factors were type 2 diabetes mellitus (15.3\% and $13.6 \%$ in SURV30 and NO-MACCE1Y, respectively), renal failure (10.8\% and $9.2 \%$, respectively), other vascular disease $(6.89 \%$ and $6.0 \%$, respectively) and IS $(2.8 \%$ and $2.4 \%$, respectively) (Table 1 ).

Prevalence of clinical characteristics in patients with and without HTR has been reported in Table S2 and Figure S1.

\section{Long-term incidence of MACCE}

The overall 5-year cumulative incidence of MACCE after AMI was higher in patients with HTR compared with those without

Table 1. Prevalence of baseline characteristics and comorbidities in survivors at 30 days (SURV30) and patients free from MACCE at 1 year (NO-MACCE1Y) from the index hospitalization for acute myocardial infarction (2009-2010).

\begin{tabular}{|c|c|c|c|c|}
\hline \multirow[b]{2}{*}{ Age (years), mean and SD } & \multicolumn{2}{|c|}{ SURV30 $(n=186,646)$} & \multicolumn{2}{|c|}{ NO-MACCE1Y $(\mathrm{n}=163,578)$} \\
\hline & 69.7 & 13.3 & 68.8 & 13.3 \\
\hline Gender (F) & 64,903 & 34.8 & 55,462 & 33.9 \\
\hline Arterial hypertension & 50,756 & 27.2 & 41,011 & 25.1 \\
\hline Dyslipidemia & 16,469 & 8.8 & 13,761 & 8.4 \\
\hline Obesity & 7095 & 3.8 & 6149 & 3.8 \\
\hline $\mathrm{PCI}$ at the index admission & 100,052 & 53.6 & 91,602 & 56.0 \\
\hline Stable coronary heart disease & 38,936 & 20.9 & 31,134 & 19.0 \\
\hline Heart failure & 40,568 & 21.7 & 31,301 & 19.1 \\
\hline Cardiomyopathies & 6437 & 3.4 & 5011 & 3.1 \\
\hline Arrhythmias & 19,379 & 10.4 & 15,112 & 9.2 \\
\hline Rheumatic heart disease & 4316 & 2.3 & 3330 & 2.0 \\
\hline Cardiac complications & 5471 & 2.9 & 4212 & 2.6 \\
\hline Peripheral vascular disease & 10,061 & 5.4 & 7984 & 4.9 \\
\hline Non-ischemic stroke or transient ischemic attack & 16,390 & 8.8 & 12,867 & 7.9 \\
\hline Anemia & 11,973 & 6.4 & 8895 & 5.4 \\
\hline Chronic obstructive pulmonary disease & 14,630 & 7.8 & 11,380 & 7.0 \\
\hline Inflammatory chronic diseases & 5192 & 2.8 & 4400 & 2.7 \\
\hline Tumors & 13,607 & 7.3 & 10,952 & 6.7 \\
\hline Previous PCI & 21,929 & 11.7 & 18,406 & 11.3 \\
\hline Previous coronary artery bypass graft & 8626 & 4.6 & 6829 & 4.2 \\
\hline Other previous cardiac surgery & 2706 & 1.4 & 2112 & 1.3 \\
\hline Previous vascular surgery & 4263 & 2.3 & 3294 & 2.0 \\
\hline Previous cerebral revascularization & 2171 & 1.2 & 1651 & 1.0 \\
\hline
\end{tabular}

MACCE, major cardiac and cerebrovascular events; PCI, percutaneous coronary intervention; values are number and percentage except where indicated otherwise. 
HTR in both SURV30 and NO-MACCE1Y (Figure 2a). The hazard ratios (HR) of 5-year MACCE for HTR and for the single risk factors constituting it, in both SURV30 and NO-MACCE1Y, are shown in Table 2. After adjusting for the general characteristics, comorbidities and cardiac procedures reported in Table 1, multivariate analyses showed an increased risk for MACCE within 5 years of $49 \%$ in the HTR SURV30 group and of $46 \%$ in the HTR NO-MACCE1Y group, compared with the patients without HTR (NO-HTR). The 5-year incidence rates (IR) of in-hospital MACCE in the HTR SURV30 and HTR NO-MACCE1Y groups were $28.5 \%$ and $16.7 \%$, respectively, with an absolute difference of $11.8 \%$. Patients treated with PCI at the index hospitalization showed less long-term MACCE than those treated conservatively in the SURV30 group [HR, 0.56; 95\% confidence interval (CI), $0.55-0.57]$ and in the NO-MACCE1Y group (HR, 0.65; 95\% CI, 0.63-0.66). In patients with HTR, however, the increase of in-hos- pital MACCE did not change after adjusting for PCI at the index hospitalization. When considered separately, all the risk factors determining HTR emerged as significant independent predictors of MACCE in both the SURV30 and NO-MACCE1Y group (Table 3 ). Although the hazard-proportionality has been respected at 5year follow-up analysis, the trend of hazard ratios for developing in-hospital MACCE in SURV30 with HTR vs SURV30 without HTR showed a time-effect in the first 18 months, with a progressive increase of the excess risk of HTR from HR $=1.15$ to $\mathrm{HR}=$ 1.50 and a subsequent stabilization (Figure $3 \mathrm{a}$ ).

\section{Long-term incidence of AMI}

The HR of 5-year recurrent AMI for HTR and for the single risk factors in both the SURV30 and NO-MACCE1Y group is shown in Table 2 . After adjusting for the general characteristics and comorbidities reported in Table 1, multivariate analyses
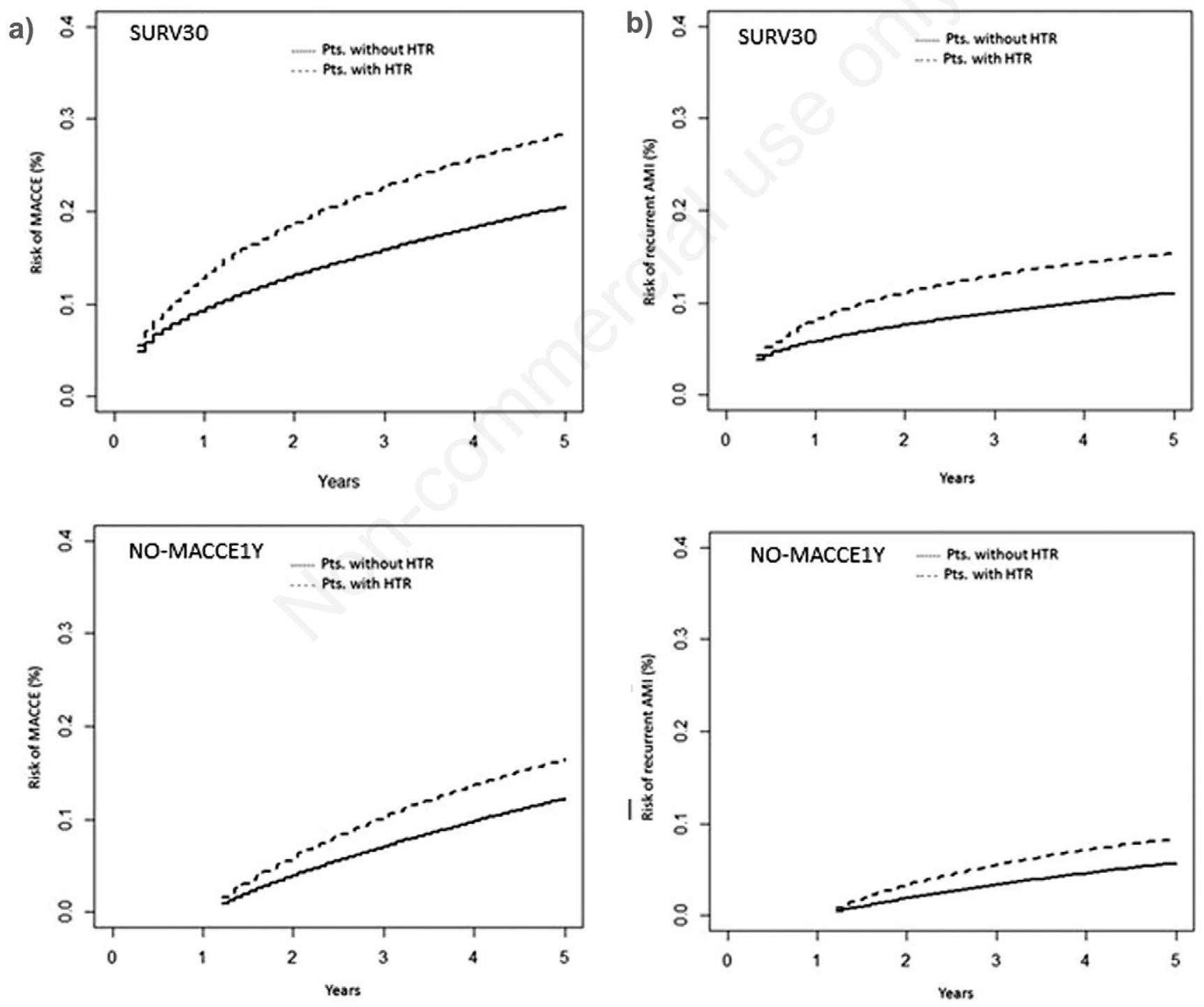

Figure 2. a) Cumulative incidence of in-hospital MACCE after AMI in patients with high thrombotic risk (HTR) (dotted line) and without high thrombotic risk (solid line) in survivors at 30 days (SURV30) and patients free from MACCE at 1 year (NO-MACCE1Y) from the index hospitalization for AMI (2009-2010). b) Cumulative incidence of recurrent in-hospital AMI after the index hospitalization for MI 2009-2010) in HTR (dotted line) and NO-HTR patients (solid line), in both SURV30 and in NO-MACCE1Y. Multivariate analysis. 
showed an increased risk of 5-year AMI of $46 \%$ in the HTR SURV30 group (IR, $15.7 \%$ ) and of $55 \%$ in the HTR NOMACCE1Y group (IR, 8.2\%), compared with the respective NOHTR patients.

The cumulative incidence of recurrent AMI was significantly higher in HTR than in NO-HTR patients, both in the SURV30 and NO-MACCE1Y group (Figure 2b) The 5-years trend of HR for developing a recurrent MI in HTR SURV30 versus NO-HTR SURV30 showed a similar time effect as that observed for in-hospital MACCE, with a progressive increase in excess risk in the first 18 months after the index hospitalization and a subsequent stabilization until the fifth year of follow-up. Anyway, also for this analysis, the hazard-proportionality at 5-year follow-up was respected (Figure 3b).

\section{Discussion}

The main finding of this study is that, after an AMI, the longterm risk of a new adverse clinical event is almost $50 \%$ higher in patients with HTR. Moreover, in contrast to what has been believed so far, the excess risk of in-hospital MACCE in HTR versus NO-HTR patients remains high up to 5 years after the index hospitalization. Although the proportional hazard analysis considered the 5-year follow-up, the trend for the HR showed a time effect with a progressive increase in the HR from time zero to 18 months, and then the curve plateaued, indicating an enduring risk of recurrent MACCE or AMI in the long term.

The present study confirms and extends a previous observation from the Swedish registry in which advanced age, stroke, diabetes, heart failure, and the lack of revascularization during index AMI were each independently associated with a higher risk of ischemic events and death [11]. The cumulative risk of recurrent cardiovascular events in that study was high in the first year after the index hospitalization and remained high in the following 36 months. In our analysis we adjusted for all the confounding factors including heart failure, a strong predictor of recurrent ischemic events but also a major predictor of mortality. This allowed us to focus on the impact of HTR alone in a contemporary population in whom the use of PCI during the index admission was even higher than in the Swedish Register (53.6\% versus $42.9 \%$ ). In our study, patients treated with PCI at the index hospitalization showed less long-term in-hospital MACCE than those treated conservatively. However, in

Table 2. Five-year MACCE and 5-year AMI adjusted hazard ratios for the high thrombotic risk and the single risk factors constituting it, in survivors at 30 days (SURV30) and patients free from MACCE at 1 year (NO-MACCE1Y) from the index hospitalization for AMI.

\begin{tabular}{|c|c|c|c|c|}
\hline \multirow[t]{2}{*}{ 5-year MACCE } & \multicolumn{2}{|c|}{ SURV30 } & \multicolumn{2}{|c|}{ NO-MACCE1Y } \\
\hline & HR & CI & HR & CI \\
\hline High thrombotic risk & 1.49 & $1.45-1.52$ & 1.46 & $1.41-1.51$ \\
\hline Type 2 diabetes & 1.37 & $1.34-1.40$ & 1.24 & $1.20-1.29$ \\
\hline Previous AMI & 1.24 & $1.21-1.27$ & 1.21 & $1.17-1.25$ \\
\hline Ischemic stroke & 1.26 & $1.21-1.32$ & 1.12 & $1.05-1.20$ \\
\hline Other vascular disease & 1.20 & $1.17-1.24$ & 1.18 & $1.13-1.23$ \\
\hline Renal failure & 1.37 & $1.34-1.41$ & 1.31 & $1.27-1.36$ \\
\hline \multirow[t]{2}{*}{ 5-year AMI } & \multicolumn{2}{|c|}{ SURV30 } & \multicolumn{2}{|c|}{ NO-MACCE1Y } \\
\hline & HR & CI & HR & CI \\
\hline High thrombotic risk & 1.46 & $1.41-1.51$ & 1.55 & $1.47-1.63$ \\
\hline Type 2 diabetes & 1.29 & $1.24-1.34$ & 1.29 & $1.22-1.37$ \\
\hline Previous AMI & 1.39 & $1.34-1.44$ & 1.45 & $1.38-1.53$ \\
\hline Ischemic stroke & 1.03 & $0.96-1.10$ & 1.00 & $0.89-1.11$ \\
\hline Other vascular disease & 1.14 & $1.09-1.19$ & 1.14 & $1.06-1.22$ \\
\hline Renal failure & 1.19 & $1.15-1.23$ & 1.24 & $1.17-1.31$ \\
\hline
\end{tabular}

MACCE, major cardiac and cerebrovascular events (hazard ratio adjusted for general characteristics and comorbidities reported in Table 1, selected by a stepwise multiple Cox regression analysis). AMI, acute myocardial infarction (hazard ratio adjusted for general characteristics and comorbidities reported in Table 1, selected by a stepwise Fine and Gray multiple regression analysis; this methodology has been used to consider death as a competing risk).

Table 3. Prevalence of single risk factors constituting high thrombotic risk in survivors at 30 days (SURV30) and patients free from MACCE at 1 year (NO-MACCE1Y) from the index hospitalization for AMI (2009-2010).

\begin{tabular}{|c|c|c|c|c|}
\hline & SURV: & $5,646)$ & NO-MACC & $63,578)$ \\
\hline & $\mathrm{n}$ & $\%$ & n & $\%$ \\
\hline Type 2 diabetes & 28,585 & 15.32 & 22,245 & 13.6 \\
\hline Previous AMI & 32,287 & 17.30 & 25,865 & 15.8 \\
\hline Ischemic stroke & 5219 & 2.80 & 3895 & 2.4 \\
\hline Other vascular disease & 12,869 & 6.89 & 9749 & 6.0 \\
\hline Renal failure & 20,185 & 10.81 & 14,980 & 9.2 \\
\hline
\end{tabular}

MACCE, major cardiac and cerebrovascular events; AMI, acute myocardial infarction. 
patients with HTR, the increase in MACCE did not change even after adjusting for PCI at the index hospitalization; in other words, the effect of HTR on prognosis was not mitigated by PCI performed at the index admission.

In the last 10 years, data from several observational studies and registries have shown a paradox in post-AMI morbidity and mortality trends, with in-hospital mortality continuing to decrease [19] and an increase in post-discharge mortality [1,20-22]. The aging population, the high prevalence of heart failure [23-27], as well as the lack of long-term adherence to evidence-based therapies $[3,28,29]$, particularly the use of statins and antiplatelet agents $[30,31]$, as well as the lack of access to secondary prevention programs [32] and cardiac rehabilitation facilities [33] have been advocated to explain this phenomenon. However, the role of ischemic risk factors as independent predictors of recurrent MACCE after discharge from an AMI is still debated. In addition,
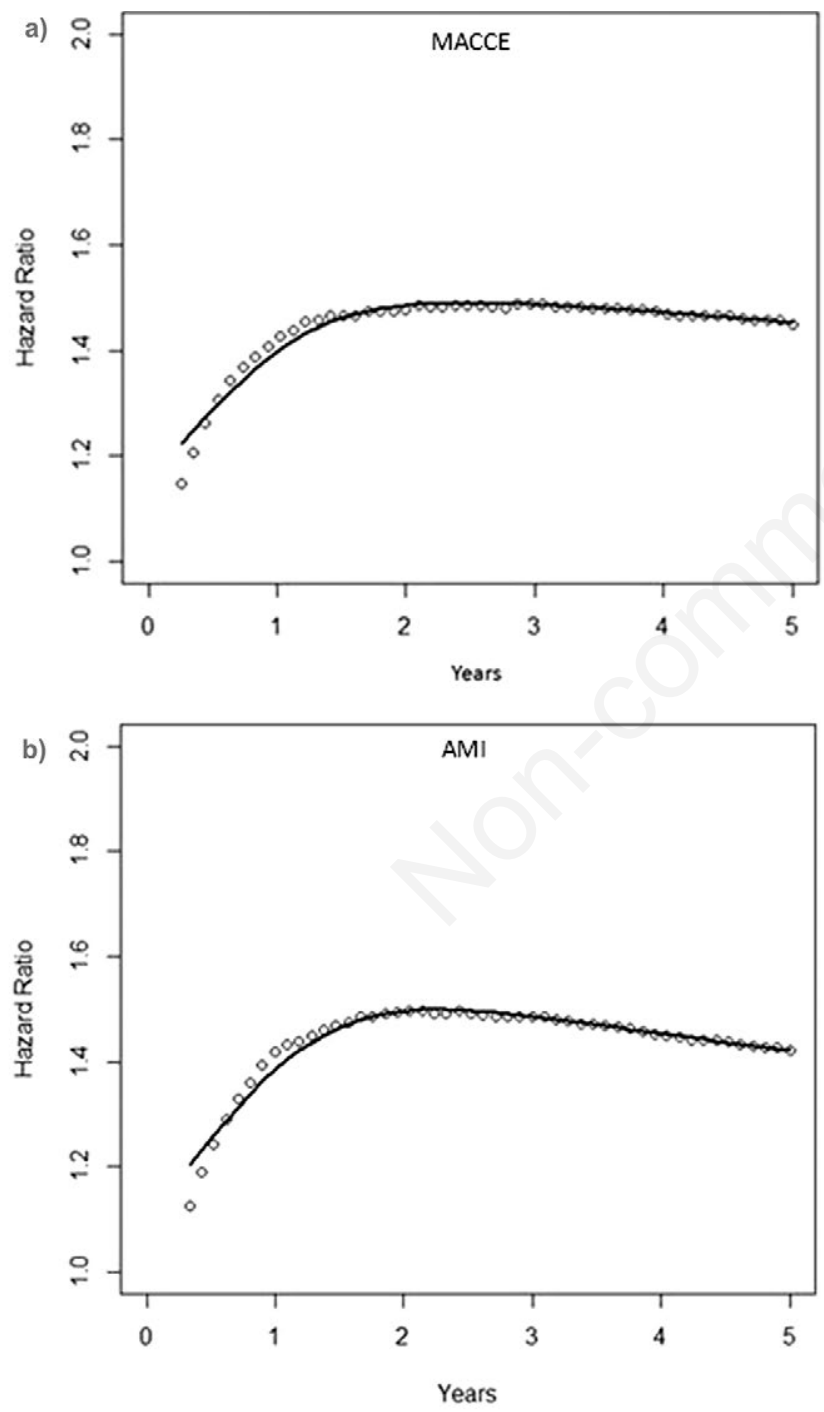

Figure 3. Five-year trend of adjusted hazard ratios of high thrombotic risk (HTR) vs NO-HTR patients for in-hospital developed MACCE (a) and AMI) (b) in survivors at 30 days (SURV30). The empty dots represent the hazard ratios point estimates and the solid line their interpolation. it is unclear if patients with HTR who have not experienced an event in the first year after AMI still have a higher risk of MACCE as compared to patients not at HTR [2].

Our data confirm the increase in long-term risk of MACCE also in HTR patients free from event in the first year after AMI. Moreover, our study suggests that the time period for which the risk appears to be increased in HTR subgroups is even longer than that reported in current clinical studies [12,13,34,35]. The excess risk due to HTR surprisingly increases up to 18 months then remains constant over time up to 5 years. One possible explanation of this finding is that adherence to therapy, including DAPT, decreases during the first year after AMI as suggested by current studies and registries [28-30,36-38]. Thus, the partial attenuation of the ischemic risk from pharmacological therapies is lost and it increases over time up to 18 months more markedly in patients with HTR. Hence, considering that the residual risk for recurrent ischemic events and re-hospitalization in patients after AMI can remain high despite optimal medical therapy, novel and/or more intense pharmacological interventions should be used over a longer period of time.

These results strongly suggest that careful selection, stratification, and long-term pharmacological management are to be warranted to this subgroup of patients, with relevant implications for organization of secondary prevention services $[32,33]$.

\section{Study limitations}

The present study is based on data from hospital discharge records. As reported in a previous work [1], although some reports have highlighted that ICD-9-CM coding adequately identifies definite or possible AMI according to the American Heart Association criteria the most common limitation of administrative data is the possible lack of strength as a basis for clinical research. Some difficulties in distinguishing between STEMI and NSTEMI must be also considered. Patients with previous AMI or ischemic stroke occurred more than 5 years before cannot be identified, and this may have led to an under-recognizing of some patients at risk. Some conditions as renal failure as defined as National Kidney Foundation stage $\geq 3$ could be underreported. Moreover, the ItHDR cannot be systematically linked to comprehensive mortality registers, thus both fatal and non-fatal events refer only to those occurred during hospitalizations. As patients discharged after AMI could also have died out of the hospital, this hindrance might be considered a limitation of the study. However, there is evidence of consistency between the reported estimates of out-of-hospital mortality in patients discharged after hospitalization due to AMI [1] and our results. Finally, we do not have information on the real use of the recommended therapies, including ACE inhibitors, aspirin, clopidogrel, $\beta$-blockers, and statins at discharge and during followup. Because decrease in adherence to therapy is frequently reported even early after ACS in European and Italian registries [28,29$33,38]$, we can assume that undertreatment in the real world can amplify the excess risk particularly in patients with HTR.

\section{Conclusions}

After an AMI, patients with HTR showed an overall higher risk of MACCE and recurrent AMI than those without HTR. This excess risk remains stable up to 5 years. Patients with HTR should be identified and treated with intensive pharmacological therapies and non-pharmacological strategies in order to improve their longterm prognosis. 


\section{References}

1. Greco C, Rosato S, D'Errigo P, et al. Trends in mortality and heart failure after acute myocardial infarction in Italy from 2001 to 2011. Int J Cardiol 2015;184:115-21.

2. Kostis WJ, Deng Y, Pantazopoulos S, et al. Trends in mortality of acute myocardial infarction after discharge from the hospital. Circ Cardiovasc Qual Outcomes 2010;3:581-9.

3. Komajda M, Kerneis M, Tavazzi L, et al. The chronic ischaemic cardiovascular disease ESC Pilot Registry: Results of the sixmonth follow-up. Eur J Prev Cardiol. 2018;25:377-87.

4. Baker DW, Einstadter SS, Husak RD, et al. Trends in post-discharge mortality and readmissions: has length of stay declined too far? Arch Intern Med 2004;164:538-544.

5. Roffi M, Patrono C, Collet JP, et al. 2015 ESC guidelines for the management of acute coronary syndromes in patients presenting without persistent ST-segment elevation: Task Force for the Management of Acute Coronary Syndromes in Patients Presenting without Persistent ST-Segment Elevation of the European Society of Cardiology (ESC). Eur Heart J 2016;37:267-315.

6. Amsterdam EA, Wenger NK, Brindis RG, et al. 2014 AHA/ACC guideline for the management of patients with non-ST-elevation acute coronary syndromes. A report of the American College of Cardiology/American Heart Association Task Force on Practice Guidelines. Circulation 2014;130:e344-426.

7. Levine GN, Bates ER, Blankenship JC, et al. 2015 ACC/AHA/SCAI focused update on primary percutaneous coronary intervention for patients with ST-elevation myocardial infarction: an update of the 2011 ACCF/AHA/SCAI Guideline for Percutaneous Coronary Intervention and the 2013 ACCF/AHA Guideline for the Management of STElevation Myocardial Infarction. J Am Coll Cardiol 2016;67:1235-50. Erratum in: J Am Coll Cardiol 2016; 67:1506.

8. Catapano AL, Graham J, De Backer G, et al. ESC Scientific Document Group2016 ESC/EAS guidelines for the management of dyslipidaemias. Eur Heart J 2016;37:2999-3058.

9. Piepoli MF, Hoes AW, Agewall S, et al. 2016 European Guidelines on cardiovascular disease prevention in clinical practice: The Sixth Joint Task Force of the European Society of Cardiology and Other Societies on Cardiovascular Disease Prevention in Clinical Practice (constituted by representatives of 10 societies and by invited experts). Developed with the special contribution of the European Association for Cardiovascular Prevention \& Rehabilitation (EACPR). Eur Heart J 2016;37:2315-81.

10. Scirica BM, Bonaca MP, Braunwald E, et al. Vorapaxar for secondary prevention of thrombotic events for patients with previous myocardial infarction: a prespecified subgroup analysis of the TRA 2P-TIMI 50 trial. Lancet 2012;380:1317-24.

11. Jernberg T, Hasvold P, Henriksson M, et al. Cardiovascular risk in post-myocardial infarction patients: nationwide real world data demonstrate the importance of a long-term perspective. Eur Heart J 2015;36:1163-70.

12. Mauri L, Kereiakes DJ, Yeh RW, et al. Twelve or 30 months of dual antiplatelet therapy after drug-eluting stents. N Engl J Med 2014;371:2155-66.

13. Bonaca MP, Bhatt DL, Cohen M, et al. Long-term use of ticagrelor in patients with prior myocardial infarction. N Engl J Med 2015;372:1791-800.

14. Bhatt DL, Fox KA, Hacke W, et al. Clopidogrel and aspirin versus aspirin alone for the prevention of atherothrombotic events. N Engl J Med 2006;354:1706-17.

15. Valgimigli M, Bueno H, Byrne RA, et al. ESC Scientific Document Group ESC Committee for Practice Guidelines (CPG), ESC National Cardiac Societies. 2017 ESC focused update on dual antiplatelet therapy in coronary artery disease developed in collaboration with EACTS: The Task Force for dual antiplatelet therapy in coronary artery disease of the European Society of Cardiology (ESC) and of the European Association for Cardio-Thoracic Surgery (EACTS). Eur Heart J 2018;39:213-60.

16. Tunstall-Pedoe H, Kuulasmaa K, Amouyel P, et al. Myocardial infarction and coronary deaths in the World Health Organization MONICA Project. Registration procedures, event rates, and case-fatality rates in 38 populations from 21 countries in four continents. Circulation 1994;90:583-612.

17. Shafazand M, Rosengren A, Lappas G, et al. Decreasing trends in the incidence of heart failure after acute myocardial infarction from 1993-2004: a study of 175,216 patients with a first acute myocardial infarction in Sweden. Eur J Heart Fail 2011;13:135-41.

18. R Development Core Team. A language and environment for statistical computing. Foundation for Statistical Computing, Vienna. Available from: http://www.R-project.org/

19. De Luca L, Marini M, Gonzini L, et al. Contemporary trends and age-specific sex differences in management and outcome for patients with ST-segment elevation myocardial infarction. J Am Heart Assoc 2016;5:e004202.

20. Krumholz HM, Wang Y, Chen J, et al. Reduction in acute myocardial infarction mortality in the United States: risk-standardized mortality rates from 1995-2006. J Am Med Assoc 2009;302:767-73.

21. Rosamond WD, Chambless LE, Heiss G, et al. Twenty-twoyear trends in incidence of myocardial infarction, coronary heart disease mortality, and case fatality in 4 US communities, 1987-2008. Circulation 2012;125:1848-57.

22. Johansen H, Brien SE, Finès $P$, et al. Thirty-day in-hospital revascularization and mortality rates after acute myocardial infarction in seven Canadian provinces. Can J Cardiol 2010;26:e243-8.

23. Bahit MC, Kochar A, Granger CB. Post-myocardial infarction heart failure. JACC Heart Fail 2018;6:179-86.

24. McManus DD, Chinali M, Saczynski JS, et al. 30-year trends in heart failure in patients hospitalized with acute myocardial infarction. Am J Cardiol 2011;107:353-9.

25. Torabi A, Rigby AS, Cleland JG. Declining in-hospital mortality and increasing heart failure incidence in elderly patients with first myocardial infarction. J Am Coll Cardiol 2009;55:79-81.

26. Kaul P, Ezekowitz JA, Armstrong PW, et al. Incidence of heart failure and mortality after acute coronary syndromes. Am Heart J 2013;165:379-85.e2.

27. Thune JJ, Signorovitch JE, Kober L, et al. Predictors and prognostic impact of recurrent myocardial infarction in patients with left ventricular dysfunction, heart failure, or both following a first myocardial infarction. Eur $\mathrm{J}$ Heart Failure 2011;13:148-53.

28. Kotseva K, Wood D, De Bacquer D, et al. EUROASPIRE IV: a European Society of Cardiology survey on the lifestyle, risk factor and therapeutic management of coronary patients from 24 European countries. Eur J Prev Cardiol 2016;23:636-48.

29. Urbinati S, Olivari Z, Gonzini L, et al. Secondary prevention after acute myocardial infarction: drug adherence, treatment 
goals, and predictors of health lifestyle habits. The BLITZ-4 Registry. Eur J Prev Cardiol 2015;22:1548-56.

30. Ho PM, Spertus JA, Masoudi FA, et al. Impact of medication therapy discontinuation on mortality after myocardial infarction. Arch Intern Med 2006;166:1842-47.

31. Khan SU, Khan MS, Kaluski E Alkhouli MA. Compliance with the prescription of recommended medical therapy in trials comparing six versus 12 months or longer dual antiplatelet therapy: A systematic review and meta-analysis. Eur J Prev Cardiol 2019;26:1673-6.

32. Schiele F, Ecarnot F, Chopard R. Coronary artery disease: Risk stratification and patient selection for more aggressive secondary prevention. Eur J Prev Cardiol 2017;24:88-100.

33. Hansen D, Piepoli MF, Doehner W. The importance of rehabilitation in the secondary prevention of cardiovascular disease. Eur J Prev Cardiol 2019;26273-6.

34. Sabatine MS, Giugliano RP, Keech AC, et al. Evolocumab and clinical outcomes in patients with cardiovascular disease. $\mathrm{N}$ Engl J Med 2017;376:1713-22.
35. Cannon CP, Blazing MA, Giugliano RP, et al. Ezetimibe added to statin therapy after acute coronary syndromes. N Engl J Med 2015;372:2387-97.

36. Udell JA, Bonaca MP, Collet JP, et al. Long-term dual antiplatelet therapy for secondary prevention of cardiovascular events in the subgroup of patients with previous myocardial infarction: a collaborative meta-analysis of randomized trials. Eur Heart J 2016;37:390-9.

37. Bittl JA, Baber U, Bradley SM, et al. Duration of dual antiplatelet therapy: a systematic review for the 2016 ACC/AHA guideline focused update on duration of dual antiplatelet therapy in patients with coronary artery disease: a report of the American College of Cardiology/American Heart Association Task Force on Clinical Practice Guidelines. J Am Coll Cardiol 2016;68:1116-39.

38. Maggioni AP, Rossi E, Cinconze E, et al. Outcomes, health costs and use of antiplatelet agents in 7,082 patients admitted for an acute coronary syndrome occurring in a large community setting. Cardiovasc Drugs Ther 2013;27:333-40. 